\title{
Electron Microscopy of Heterostructure for Solar Energy Recovery: ZnO Nanowires and $\mathrm{Co}_{3} \mathrm{O}_{4}$ Nanoparticles
}

\author{
O. Cigarroa-Mayorga ${ }^{1}$, E. Neri ${ }^{1}$, H. A. Calderon ${ }^{1}$ and C. Kisielowski ${ }^{2}$. \\ 1. Depto. Física, Instituto Politécnico Nacional - ESFM, UPALM Zacatenco Ed. 9, CDMX, Mexico. \\ 2. Molecular Foundry-NCEM, LBNL, Berkeley CA, USA.
}

Heterostructures consisting of nanowires and nanoparticles are important components of solar energy recovery devices. These heterostructures can be used to decompose water into $\mathrm{H}_{2}$ and $\mathrm{O}_{2}$ as a first step to fabricate a fuel (light hydrocarbon) with the help of solar energy. A complementing stage is to reduce $\mathrm{CO}_{2}$ and combine the product with $\mathrm{H}_{2}$ to produce a liquid fuel. The heterostructure has an array of nanowires made of a semiconductor material to capture solar light. Additionally, attached nanoparticles act as catalysts for the water decomposition reaction. In this investigation, nanowires are targeted to be $\mathrm{ZnO}$ and nanoparticles of $\mathrm{Co}_{3} \mathrm{O}_{4}$. The nanowires have been synthesized by means of electrochemical deposition on ITO and by using an alumina template. Nanoparticles (NPs) have also been electrolytically deposited directly on the nanowires array and independently by mechanical milling. The alumina template is synthesized by using pure $\mathrm{Al}$ and a two-step anodizing process [1]. The alumina template is used to electrodeposit $\mathrm{ZnO}$ by means of a potentiostat with a potential difference of $-1 \mathrm{~V}$ and a calomel reference electrode at $70{ }^{\circ} \mathrm{C}$ and for 1,30 and $60 \mathrm{~min}$. Then the template is dissolved in $\mathrm{NaOH}$. Nanoparticles have been independently synthesized by reactive chemical milling and also by electrochemical deposition. This last technique is used to assemble the heterostructure by using Co deposition directly on the nanowire array in a potentiostat and then oxidizing in air at $450{ }^{\circ} \mathrm{C}$.

The components of the heterostructure have been characterized by means of electron microscopy and other techniques. Figure 1a shows a SEM image of an alumina template used for electrochemical deposition of the nanowires. The side view (Fig. 1b) shows a template with possible wire lengths close to $5 \mu \mathrm{m}$. The as deposited array of $\mathrm{ZnO}$ nanowires is given in Fig. 1c. The complete heterostructure i.e., the array of nanowires together with the NPs, is shown in Fig. 1d. The nanowires length is approximately $5 \mu \mathrm{m}$ with a diameter close to $50 \mathrm{~nm}$ and with NPs sizes ranging between 2 and $10 \mathrm{~nm}$. This corresponds to a deposit time of $30 \mathrm{~min}$. Nanoparticles are shown in Figure 2. A rather low dose rate has been used to record 40 images at different defoci and apply an exit wave reconstruction process by means of MacTempas ${ }^{\circledR}$. The corresponding phase image is given in Fig. 2a for a dose rate of $20 \mathrm{e}^{-}$ $/ \AA^{2}$ s. Here the structural characteristics of $\mathrm{Co}_{3} \mathrm{O}_{4}$ can be recognized together with a rather limited long range order. This phase image corresponds very likely to a genuine structure of the nanoparticle after synthesis. Exposing the NP to the beam for longer times and higher dose rates makes possible to change the atomic distribution and ordering of the nanoparticle. This is shown in Fig. $2 \mathrm{~b}$ where the dose rate is again $20 \mathrm{e}^{-} / \AA^{2} \mathrm{~s}$ but this time after acquiring 40 images at $1000 \mathrm{e}^{-} / \AA^{2} \mathrm{~s}$. The effects on the image and the corresponding diffraction pattern (insets) are clearly affecting the atomic distribution and the ordering [3].

References:

[1] X. Liu et al, Ceramics International 41 (2015), p. 11710.

[2] O. Cigarroa Mayorga. Master Thesis Dissertation No. A150806, IPN, Mexico. 2016.

[3] The authors acknowledge funding from Instituto Politécnico Nacional (COFAA, SIP) and CONACYT

through grants FOINS 75/2012 and CIAM 148304. 6]. Work at the Molecular Foundry is supported by the Office of Science, the Office of Basic Energy Sciences, the U.S. Department of Energy 
under Contract No. DE-AC02-05CH11231.

(a)

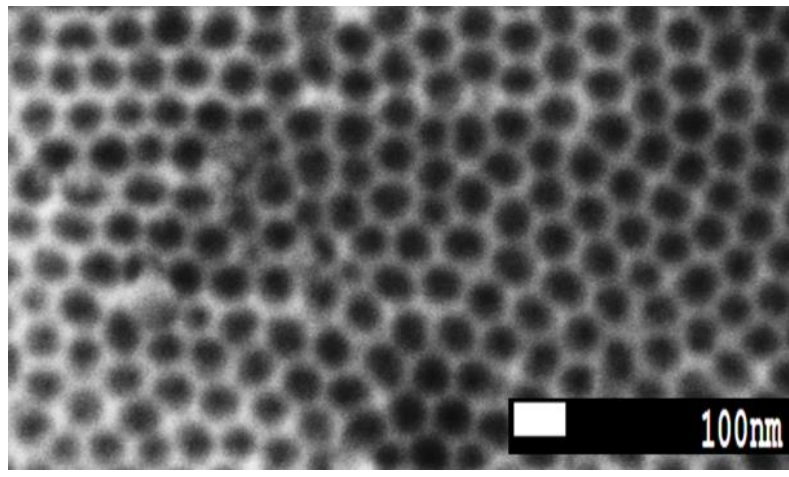

(c)

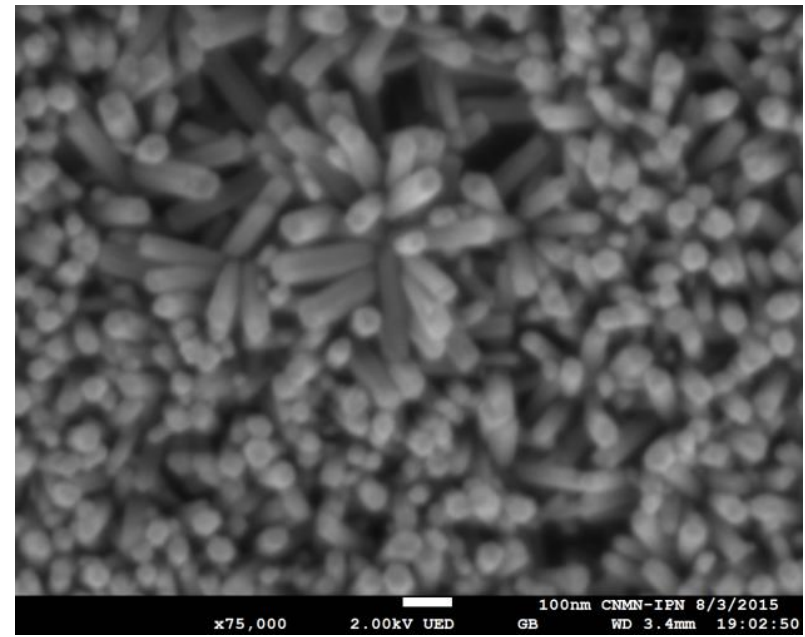

(b)

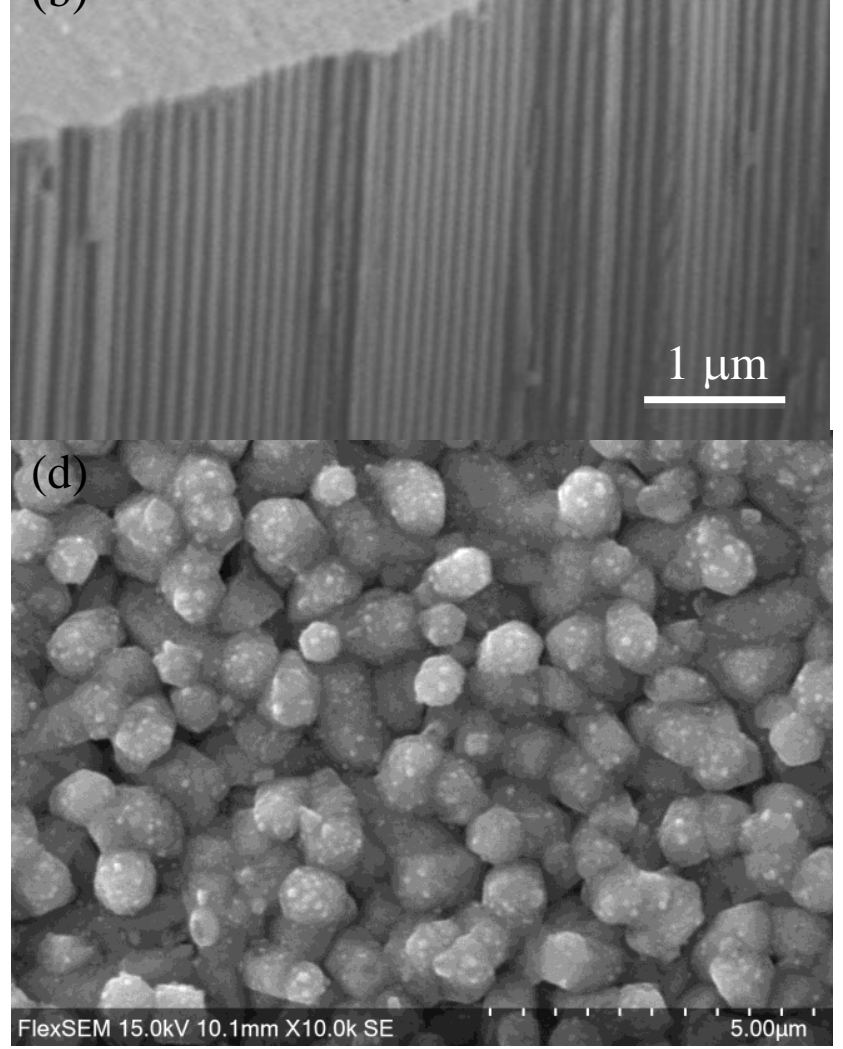

Figure 1. ZnO nanowire array. (a) Alumina template with array of holes to deposit the nanowires. (b) Alumina template side view. (c) Nanowires array after $30 \mathrm{~min}$ of electrochemical deposit. (d) Heterostructure with $\mathrm{ZnO}$ nanowires and $\mathrm{Co}_{3} \mathrm{O}_{4}$ nanoparticles.

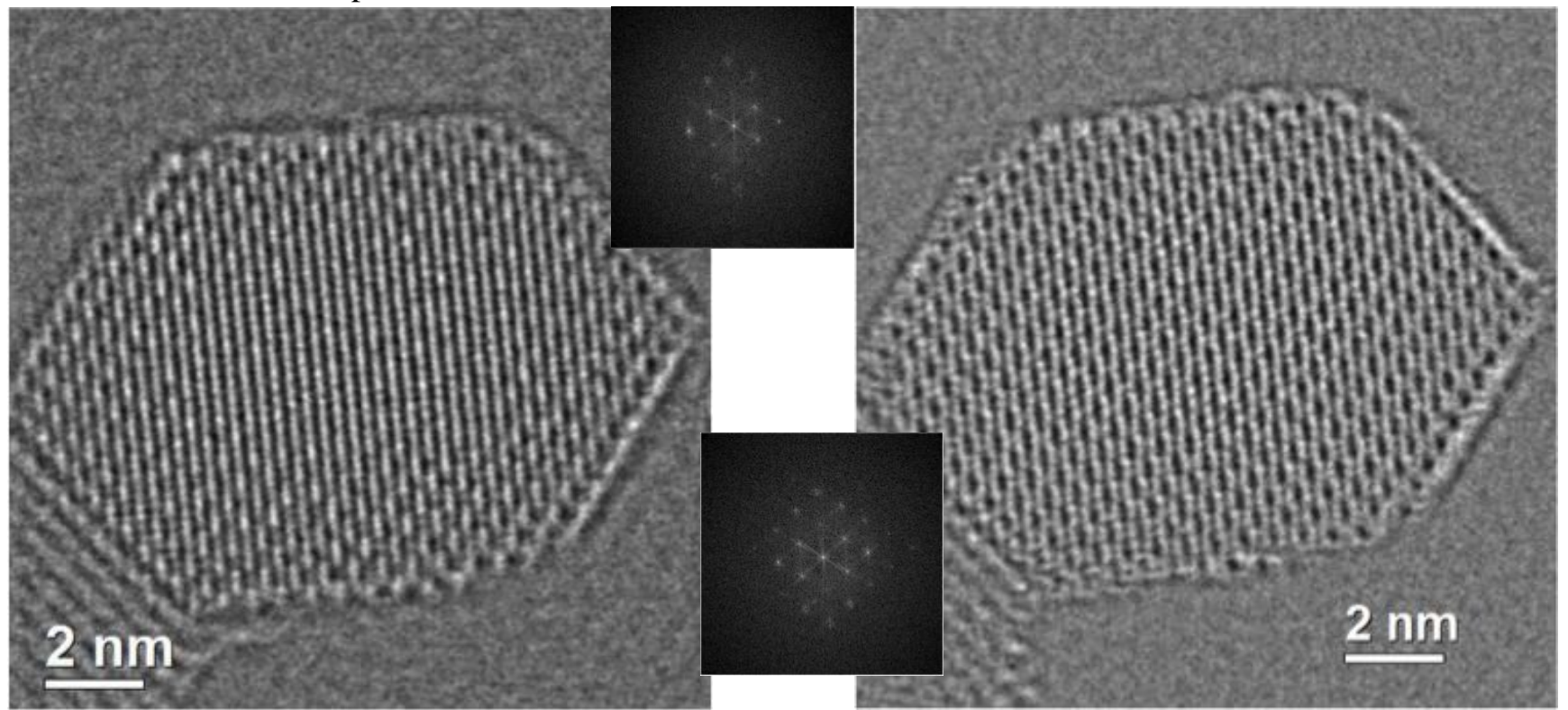

Figure 2. $\mathrm{Co}_{3} \mathrm{O}_{4}$ nanoparticles in the heterostructure. (a) Low dose rate phase image after EWR procedure. (b) Phase image after exposure of particle to higher dose rates. Insets show the corresponding diffraction patterns. 